\title{
La universidad en su redefinición epistemológica institucional
} The University and its epistemological, institutional redefinition A universidade em sua redefinição epistemológica institucional

\author{
Carolina Cortez Schall \\ Universidad Nacional Tres de Febrero \\ Buenos Aires, Argentina \\ schallcarolina@hotmail.com \\ (D) https://orcid.org/ 0000-0003-1442-8780
}

Recibido - Received - Recebido: 02 / 11 / 2020 Corregido - Revised - Revisado: 12 / 03 / 2021 Aceptado - Accepted - Aprovado: 14 / 04 / 2021

https://doi.org/10.22458/ie.v23i34.3299

URL: https://revistas.uned.ac.cr/index.php/innovaciones/article/view/3299

\begin{abstract}
Resumen: En este ensayo se aborda, como objeto de estudio, la definición de la Universidad Argentina y sus funciones desde sus orígenes, a través de una mirada analítica, focalizada en la transformación de la ciencia, la tecnología y su vínculo con el medio socio-productivo. Para luego concluir sobre las situaciones sociales emergentes que establecen la redefinición de las funciones de la universidad, visibilizadas en sus políticas, estrategias y prácticas concretas; en primer lugar,enlazadas a resolver las problemáticas sociales, para luego llegar al sector productivo y económico de un país.Frente a una transformación continua y dinámica de las estructuras configurantes de la universidad que se conoce, se evidencia un proceso de constantes cambios sociales y culturales, los cuales demandan otras funciones y acciones de las instituciones enmarcadas en un contexto político, social y económico; en medio de este, indefectiblemente la universidad, además de buscar su propio financiamiento, debe vincularse por medio de la empatía y la responsabilidad social.

La situación emergente que se suscita, de una profunda crisis social, económica y sanitaria, lleva ante la inminente necesidad de pensar y repensar en la generación de políticas desde la gestión universitaria, que le ofrezcan centralidad y sustento al conocimiento para el desarrollo y el acompañamiento actual y futuro de la sociedad. Todo esto, por medio de la innovación, la tecnología y la transferencia de la ciencia de la mano con el medio socio productivo desde una profunda convicción de responsabilidad y calidad social universitaria.
\end{abstract}

Palabras Clave: Universidad, Responsabilidad social, investigación, Tecnología, políticas universitarias

Summary: This essay addresses the definition of University in Argentina and its functions from its origins. This is done through an analytical view on the science and technology transformations and their connection to the socio-productive sector. It describes the emergent social situations that establish the redefinition of the University's functions, made visible at the hand of its politics, strategies as well as concrete practices. Firstly, aiming at solving social issues, and then reach the country's productive and economic sectors.

Faced with a continuous and dynamic transformation of the known configuration structures of the University, a process of constant social and cultural changes is evident, which demands other functions and actions from the institutions framed in a political, social, and economic context. In the midst of this, unfailingly, the University, in addition to seeking its own funding, must be linked through empathy and social responsibility.

Due to a profound social, economic, and health crisis, the emerging situation leads to the imminent need to think and rethink the generation of university management policies. This offers centrality and support for knowledge 
development and will bolster the present and the future of society. This will be carried out through innovation, technology, and science transfer hand in hand with the socio-productive sector from a deep conviction of responsibility and University social quality.

Keywords: University, social responsibility, research, technology, University policies

Resumo: Este ensaio aborda, como objeto de estudo, a definição da Universidade Argentina e suas funções desde suas origens, através de um olhar analítico, voltado para a transformação da ciência, da tecnologia e sua ligação com o ambiente socioprodutivo. Em seguida, concluir sobre as situações sociais emergentes que estabelecem a redefinição das funções da universidade, visíveis em suas políticas, estratégias e práticas concretas; em primeiro lugar, ligadas à solução de problemas sociais, para depois alcançar o setor produtivo e econômico de um país. Diante de uma contínua e dinâmica transformação das estruturas que configuram a universidade conhecida, é evidente um processo de constantes mudanças sociais e culturais, que exigem outras funções e ações das instituições enquadradas em um contexto político, social e econômico; em meio a isso, a universidade, além de buscar seu próprio financiamento, deve estar ligada por meio da empatia e da responsabilidade social.

A situação emergente, de uma profunda crise social, econômica e sanitária, leva à necessidade iminente de pensar e repensar na geração de políticas a partir da gestão universitária, que oferecem centralidade e apoio ao conhecimento para o desenvolvimento e o acompanhamento atual e futuro da sociedade. Tudo isso, através da inovação, da tecnologia e da transferência da ciência de mãos dadas com o ambiente socioprodutivo a partir de uma profunda convicção de responsabilidade social universitária e qualidade.

Palavras chave: Universidade, responsabilidade social, pesquisa, tecnologia, políticas universitárias

\section{INTRODUCCIÓN}

Como objeto de estudio, en este trabajo se pretende abordar la definición de la Universidad y sus funciones en el contexto argentino. Desde sus orígenes a través una mirada analítica y focalizada en la transformación de la ciencia, la tecnología y su vínculo con el medio socio-productivo. Para luego concluir sobre las situaciones sociales emergentes que establecen la redefinición de las funciones de la universidad, visibilizadas en sus políticas, estrategias y prácticas concretas, enlazadas a resolver las problemáticas sociales en primer lugar, para luego llegar al sector productivo y económico del país.

La universidad como entidad, históricamente marca un antes y un después en el desarrollo humano, sea en la cultura que sea. He aquí la razón e inexorable necesidad de contar con un saber reflexivo sobre el ser universitario, sus raíces, influencias, necesidades $y$, sobre todo, las responsabilidades inherentes que conlleva la formación de futuros ciudadanos profesionales; responsables del porvenir social, económico, político y cultural de una nación. Por lo anterior, este escrito persigue el fin de retomar conceptualizaciones, disposiciones reglamentarias y la retórica institucional fundante de la ideología de la Educación Superior en Argentina, para su revaloración con una mira proyectiva a un mundo aún ignoto.

\section{DESARROLLO}

\section{La universidad y sus políticas}

Se comenzará por el objeto de estudio el cual es la esencia de la vida universitaria desde sus inicios, así como su finalidad y, no debía ser otra que:

[...] la investigación y transmisión o docencia de la verdad en un nivel superior de ciencia o de-velación de sus causas en todas sus manifestaciones particulares y en su visión sapiencial de la Filosofía y de la Teología cristianas en la actual providencia del hombre que las integra o unifica en el plano fundamental de sus últimas causas. (Derisi, 1980, p. 20). 
Bajo este concepto de universidad, descrito en la cita anterior, se puede pensar a esta desde un plano netamente filosófico-religioso que deja velar una intencionalidad y empírea única. Como expone Francisco Muscará (2014), la universidad no es una institución ordenada a la acción; sino que su naturaleza resulta eminentemente teorética o especulativa. Por lo tanto, el carácter académico propio de los estudios universitarios consiste en que los distintos sectores de la ciencia y la técnica se realicen con un deseo desinteresado en descubrir y encontrar la verdad; así como también en el obrar moral y en toda la actividad práctica humana. Desde este lugar la universidad considera y decide cuáles deben ser los principios que guíen esas acciones.

El espíritu de la tarea universitaria pasa más por el estudio de la economía, las técnicas, la política, entre otros conocimientos, sin ser un centro de aplicación directa de estos. Solo en esa actitud teorética se encuentra la independencia respecto a cualquier fin utilitario $y$, de esta manera, puede fundarse una "libertad académica" (Muscará, 2014, p. 20).

Por esto se afirma que:

La Universidad es la ciencia que busca la sabiduría y el movimiento hacia la unidad (versus unum) es idéntico con ella; luego, toda la vida académica es, en el fondo, un 'versus sapientiam', hacia la Sabiduría, porque ésta se sigue de la contemplación de toda Verdad [...] La Universidad, en cuanto comunidad académica, se orienta hacia la contemplación. (Canturelli, 1964, p. 54).

A esta universidad filosófica-religiosa le siguió la universidad profesionalista, en esta se denota claramente el cambio de finalidad; pues el interés ya no pasaba por el aprender, sino primaba el alcanzar un título habilitante para el trabajo profesional. Esto se confirma en las palabras de Francisco Muscará (2014), al asegurar que: "la universidad argentina está principalmente orientada a la formación de profesionales" (p. 52). Por lo tanto, la Educación Superior en Argentina tuvo un fuerte influjo desde las instituciones educativas internacionales y se abocó a la reproducción de saberes como función vital de la formación académica, lo cual dejaba en desvalor al tratamiento de las necesidades y las problemáticas desde lo regional y social identitario. El desarrollo científico y económico quedó insignificante, al igual que el trabajo interdisciplinario entre cátedras, unidades académicas e instituciones.

Por otro lado, la política dentro de la gestión universitaria ha ido desarrollándose en forma cambiante y de manera sostenida en el tiempo, a partir de la finalidad que ha perseguido. Este proceso ha evolucionado en concordancia al cómo ha ido mutando la definición de las funciones universitarias para con el medio social.

La tarea residía en pensar en políticas universitarias, al partir desde la definición y la redefinición de la universidad, desde un lugar "no cientificista" como se postularon las universidades europeas durante el lluminismo. Estas presentaban menos importancia en ciencias en contraste a las academias científicas, tales como la Academia Francesa de Ciencias (Jacovkis, 2019). El desarrollo científico fue tomando relevancia con la ayuda de las escuelas de medicina en universidades escocesas. También las instituciones alemanas colaboraron con este desarrollo (Comisión Europea, 2012; Maroto Barquero, 1989; Pascolini, 2012).

Según Pastor, (2018) y López Muñoz, (2020) establecen, sobre todo, la universidad Göttingen, que desde una concepción liberal, reforzaba la investigación y la publicación del conocimiento (Böhme, 2021). De esta forma, las academias promovidas desde la Revolución Científica como impulsoras del progreso científico en contraste al escolasticismo que constituían las universidades se posicionaron desde un lugar emergente en el desarrollo de la ciencia y la generación de conocimiento científico.

Este movimiento originó a las sociedades científicas no oficiales, conferencias públicas con acceso del género femenino antes vetado, revistas científicas, el surgimiento de enciclopedias, entre otros. El posicionamiento sobre el conocimiento como un valor de poder necesario para el desarrollo y el crecimiento 
de un grupo social, era un devenir que día a día tomaría mayor relevancia y peso junto con el avance de la tecnología.

Las actividades científicas que se venían desarrollando solamente en las Grandes Écoles, tuvieron acogida en la universidad recién a partir de 1896, cuando la estructura única que sobrevivió en Francia, bajo un fuerte control del Estado, se dividió en 17 universidades.

Por otro lado, el sistema humboldtiano favoreció la investigación en forma profesional, la reguló burocráticamente y con laboratorios bien equipados. Este sistema se convirtió en el responsable del desarrollo de una universidad moderna en investigación y se posicionó sobre la idea de "libertad de investigación científica, enseñanza y estudio", la cual perduró hasta 1945 (Jacovkis, 2019).

La influencia de este sistema alemán tuvo alcance sobre las universidades europeas, lo cual colaboró en el éxito de las actividades de investigación en ciencias naturales e ingeniería. Sin embargo, este modelo llevaba a las universidades bajo un sistema predominantemente competitivo y elitista. Argentina no quedó eximida de esta condición, en palabras de Pérez Lindo (1985): "Hacia 1960 las concepciones de la Universidad comenzaron a replantearse en todo el mundo" (p. 121). Este fenómeno se suscita por la abundante producción y proliferación científica, a la cual la UNESCO denominó como "explosión de conocimientos". La aplicación sistemática y masiva de la ciencia y la técnica, llevaba a soñar de nuevo con el progreso indefinido que los iluministas y positivistas imaginaban en el siglo XIX.

\section{Universidad y ciencia}

Así como el proyecto Manhattan fue el primer emprendimiento en investigación y desarrollo (Coronado, 2005; Luque Cózar y Domínguez López, 2009; Vergara Aimone, 1997), durante la segunda guerra mundial, la creación de la primera computadora que puede considerarse como tal, sucede en 1938 en manos del ingeniero Konrad Suze (Santos, 2018). Esto marca un hito en la historia de la ciencia y la tecnología a nivel mundial. Diversas universidades desarrollaron este proyecto tecnológico; impulsó el trabajo articulado entre investigación de nuevos procesos y sistemas, el desarrollo tecnológico y la innovación que marcaría el comienzo de una sociedad caracterizada por el avance del conocimiento científico.

En este sentido en Argentina, bajo la presidencia de Sarmiento, se crea la Academia Nacional de Ciencias, con la Ley 322 el 11 de setiembre de 1869 en Córdoba (Tognetti y Page, 2000). Esta misma ley autorizaba al Poder Ejecutivo a contratar dentro o fuera de Argentina hasta veinte personas docentes, quienes serían destinadas a la enseñanza de ciencias especiales en la Universidad de Córdoba y en los colegios nacionales.

En 1870 comenzaron a establecerse las primeras personas docentes extranjeras, provenientes en su mayoría de Alemania. El personal contratado por el gobierno, tenía la responsabilidad de formar un cuerpo docente en ciencias naturales y exactas, así como de llevar a cabo la investigación científica del territorio nacional.

Luego, en 1878, el Poder Ejecutivo aprobó por decreto el reglamento de la Academia Nacional de Ciencias, que le dio su forma definitiva como corporación científica, separada de la Universidad Nacional de Córdoba y liberándola de las responsabilidades docentes en esta última entidad. De esta forma, se orientó al desarrollo y la divulgación de las ciencias exactas y naturales, al estudio y exploración del territorio del país, así como a asesorar al Gobierno y otras instituciones científicas, en temas de su especialidad.

A partir de 1955 la exploración científica de Argentina se trasladó a otras dependencias del Estado, como: el Consejo Nacional de Investigaciones Científicas y Técnicas (CONICET), el Instituto Nacional de 
Tecnología Agropecuaria (INTA) y la Comisión Nacional de Actividades Espaciales (CONAE). Al tiempo que se preservaba la ideología de insertar ciencia y tecnología en la trama del desarrollo, esto implicaba el saber cómo y dónde innovar, lo cual demostraba el resultado de la acción conjunta entre la Universidad, la Industria y el Estado como un hacer naciente en Argentina (Academia Nacional de Ciencias, 2020).

\section{Universidad y gestión del conocimiento}

Para la UNESCO (2010) una sociedad apoyada por su diversidad y sus capacidades, es una que se basa en el conocimiento, la cual debe promover la participación activa sobre la generación y transmisión del conocimiento, la tecnología de la información y las comunicaciones. Una característica de este tipo de sociedad es más abarcadora que las sociedades de la información; pues se puede ofrecer un enfoque nuevo y relevante para el desarrollo de una comunidad.

A partir de la década del '90, comienza a develarse y constituirse este concepto de "sociedad del conocimiento" sobre el que se despliega un sinfín de estudios que profundizaban sobre este término (Almenara, 2008; Armengol, 2005; Balderas, 2009; Bori et al., 2002; Burch, 2005; Cabero; Carranza, 2007; García Aretio, 2012; García-Peñalvo, 2014; Krüger, 2006; Mateo, 2006; Millán, 2001; Moreno, 2009; Pablos Pons, 2010; Salazar-Gómez et al., 2018; Tobón et al., 2015; Tubella et al., 2005; entre otros).

De acuerdo con lo anterior, comienza a definirse lo que se constituye como la "gestión del conocimiento en la universidad" (Abásolo, 2008; Acosta, 2011; Alonso Arévalo, 2007; Buitrago et al., 2006; Fainholc, 2006, Farfán; Jover et al., 2006; Rodríguez Castellanos et al., 2001; entre otros). Burton Clark (1991) sostiene que las sustancias de la Educación Superior son totalmente distintas de las que se encuentran en las organizaciones industriales, las oficinas gubernamentales y las diversas agencias civiles de carácter no lucrativo. Por esto mismo, se expone a Pérez Lindo (2014) al aseverar que, mientras en la empresa la inquietud principal es la gestión de los recursos humanos y materiales de manera inteligente; en la universidad todo está vinculado a modelos de conocimiento, lo que se quiere y cómo se quiere mostrar. Desde un enfoque dialéctico, se propone para "convertir los conocimientos" de lo oculto a lo explícito entre los diversos actores y niveles de la organización, superar el modo occidental de pensar con antinomias (objetivismo-subjetivismo, racionalismo-empirismo; realismo-idealismo). Esto es, para que los avances que se producen a nivel dialéctico a partir de posiciones que pueden ser contradichas, puedan dar lugar a un nuevo acuerdo sobre la realidad y así sucesivamente.

Por lo anterior, se puede aseverar que toda teoría que constituya un orden orgánico y administrativo, se encuentra asentada en modelos de conocimiento que necesariamente deben ser evidenciados, lo cual conduce el partir de un conocimiento subyacente a uno relevado en forma explícita. Y aquí es cuando la investigación toma protagonismo en esta asignación de importancia en los procesos del conocimiento del mundo productivo, al relevar las potencialidades epistémicas de este último. Una característica inherente a la universidad, es la de generar nuevas teorías y, por lo tanto, esta debe validarlas también dentro del sector productivo.

La tendencia mundial se configura hacia la función en que la universidad debe realizar actividades creativas e innovadoras, de producción y servicio, de cooperación con organismos públicos, privados y organizaciones no gubernamentales; lo cual se alinea con el posicionamiento de Burton Clark (1991), en cuanto al surgimiento de una nueva universidad desde un rol emprendedor.

Resulta oportuno destacar que las universidades e institutos de investigación modifican los modelos de generación de conocimiento y transferencia al medio, en consonancia con el avance de la sociedad y su gestión. Esta evolución ha interferido en la enseñanza universitaria, la cual se encuentra cada vez más 
involucrada con las necesidades sociales, esto obliga a romper con los compartimentos monodisciplinarios en favor de la transdisciplinariedad.

La gestión del conocimiento debe atender a las múltiples dimensiones para abordar las diversas problemáticas sociales. La coherencia entre las acciones planificadas con base en los objetivos que se persiguen, con el fin último de la administración y la gestión universitaria, debería ser el cimiento estructural que dé sustento y congruencia al marco institucional.

El conocimiento como poder llevó a que las universidades buscaran desarrollar la producción de conocimiento y de personas altamente calificadas, como medio indispensable en el avance del desarrollo económico de un país, por lo cual, esto demuestra un proceso de mayor complejidad frente a la construcción de una sociedad del conocimiento.

La universidad no solo debe procurar la génesis del conocimiento, sino además legitimarlo y divulgarlo. Esto la lleva a posicionarse desde diversos lugares, de acuerdo con las lógicas e ideologías regionales, culturales, sociales, educativas, entre otras; siempre se concibe como una entidad visionaria, gestora de cambios y articuladora con la tradición académica.

Como asegura Pérez Lindo (2014), la cultura universitaria da lugar al desarrollo de las subjetividades, la producción o reproducción de lenguajes y los intercambios simbólicos. Las concepciones mono disciplinares imponen visiones que desdibujan y deforman el mundo.

Ciertamente una concepción fundada en una visión compleja del conocimiento (Morín y Ruíz, 2005) dará prioridad a una mirada multidimensional de los aspectos relevantes para el análisis de cada fenómeno atravesado por la interdisciplinariedad, la transdisciplinariedad y la multidisciplinariedad.

\section{Universidad y políticas tecnológicas}

Se considera relevante partir del aporte de Lino Barañao:

Está claro, en este sentido, que la ciencia y la tecnología en los países de América Latina no sólo deben promover la competitividad de su sector productivo sino que, además, tienen una demanda preexistente que es la de promover la inclusión social incorporando a la ciudadanía en la apropiación de las nuevas tecnologías, lo que llamamos innovación inclusiva. Este concepto constituye uno de los ejes centrales en los que se está desarrollando el pensamiento actual en materia de ciencia y tecnología latinoamericana. (prólogo a Sábato, 2011, p. 16).

Este pensamiento sobre ciencia y tecnología sentó las bases ideológicas de los caminos a seguir en la construcción de políticas que ofrezcan respuesta al bienestar de un grupo social. No obstante, la superación en el desarrollo de América Latina, resultará de la acción simultánea de diferentes políticas y estrategias. En todo caso, el acceso a una sociedad moderna, lo cual es uno de los objetivos que se pretenden alcanzar para el desarrollo, supone necesariamente una acción decisiva en el campo de la investigación científico-tecnológica, lo cual la convierte en una poderosa herramienta de transformación de una sociedad (Sábato y Botana, 1970).

Ya en planteos prospectivos de orden mundial para este siglo, se tomaban argumentos para analizar la imperiosa necesidad de un vigoroso desarrollo científico-tecnológico en América Latina. Entre estos argumentos se exponía la relevancia de absorber las tecnologías que todo país debe importar para propiciar este desarrollo, para esto se debía contar con una sólida infraestructura científico-tecnológica, con el fin de que su resultado fuera más eficiente. 
En Argentina, las políticas tecnológicas (Chudnovsky y López, 1995) se vieron favorecidas por la apertura de la economía a las importaciones, lo cual estimularía una mejora en la eficiencia del sector productivo por medio de la mayor competencia en el mercado local; al mismo tiempo facilitaría el acceso a maquinaria y equipamiento de última generación; así como la desregulación de los acuerdos de transferencia de tecnología sería otro estímulo al proceso de modernización tecnológica.

Todo lo anterior, enmarcado en una serie de acontecimientos generados dentro de políticas para la regulación y la promoción tecnológica regional. Como fueron la Ley 23.877 de "Promoción y Fomento de la Innovación Tecnológica" reglamentada en 1992 (Ministerio de Justicia y Derechos Humanos, 1990), la cual establece un fondo de promoción con una asignación anual y crea la figura de unidades que vinculan al sector privado e institutos de investigación. También el "Programa de Modernización Tecnológica" (Ministerio de Educación, Ciencia y Tecnología, 2006) lanzado por el Ministerio de Economía bajo un préstamo del Banco Interamericano de Desarrollo (BID), destinado a dos líneas de crédito para la innovación tecnológica. Junto a la reestructuración del Instituto Nacional de Tecnología Industrial (INTI) y programas de extensionismo industrial para Pequeñas y Medianas Empresas (PyMEs) (Dini et al., 2011; Ferraro y Goldstein, 2011) de "Desarrollo de Proveedores y creación del Sistema Nacional de Normas, Calidad y Certificación" (en el ámbito de la Secretaría de Industria).

Este emprendimiento presentaba fuertes críticas con respecto a una implementación desarticulada de estas acciones; por lo tanto, carecían de un camino a seguir claro y determinado en línea con las políticas tecnológicas. Por otro lado, no fueron resueltas las demandas tecnológicas y de organización para el desarrollo de la competitividad sistémica del sector manufacturero, y en especial de las PyMEs. Sobre este escenario, la crisis se profundizó en las viejas instituciones y corrieron el riesgo de quedar en partidas obsoletas en cuanto a la planificación estratégica tecnológica.

En consonancia con lo anterior descrito, surge el concepto de "parque industrial" (Lanzafame, 2019; Venturuzzi et al., 2007), cuyo objetivo propuesto, tanto en Argentina como en otros países, fue el de contar con un instrumento de desarrollo económico y diversificación industrial, lo cual acarreaba un aumento del empleo y, por otro lado, se concebía como un elemento de ordenamiento urbanístico. Estos parques se diferencian del tejido urbano habitacional o del espacio periurbano en el que se inserta y su objetivo sería la creación de economías a escala, con la posibilidad de ofertar espacios, servicios y calidad específicamente adaptados a las necesidades de las industrias.

Por su parte, la Organización de las Naciones Unidas para el Desarrollo Industrial (ONUDI), categoriza a las áreas urbanas especialmente dedicadas a las industrias en: Parques Industriales, Zonas Económicas Especiales, Eco Parques Industriales, Parques Tecnológicos y Distritos de innovación. Estos espacios se vinculan con el nivel de desarrollo económico de un país (Fundación Metropolitana, 2014).

Durante el 2020 en Argentina, en la búsqueda de dar mayor impulso a estos parques, se crea el Programa Nacional para el Desarrollo de Parques Industriales, desde el Poder Ejecutivo Nacional. Lo anterior con el objeto de continuar profundizando la implementación de políticas públicas que promuevan el desarrollo de las economías regionales. También así, se busca incentivar el ordenamiento territorial y la planificación productiva local, así como maximizar la integración dentro de un determinado ámbito espacial, atraer inversiones nacionales y extranjeras, aumentar las oportunidades de generación de empleo y garantizar la sostenibilidad de las actividades económicas. Y como consecuencia, se persigue el ampliar el universo de estos parques industriales registrados, para fortalecer las capacidades de los gobiernos locales sobre la gestión de proyectos, planificación de las inversiones públicas y la contribución al desarrollo económico y social del país (Inteligencia Jurídica, 2020).

Por otro lado, el uso inteligente de los recursos naturales, las materias primas, la mano de obra y del capital, como los problemas de las economías de escala, como se expuso anteriormente, se requieren en formas específicas para cada país. También la transformación de las economías para satisfacer la 
necesidad imperiosa de industrialización y exportación de productos manufacturados, irá atravesado acorde al potencial científico-tecnológico del territorio.

En ese contexto, nace el Banco Nacional de Proyectos de Desarrollo Tecnológico y Social (PDTS) como estrategia para orientar las actividades del sistema científico argentino a la resolución de problemas de la sociedad, el medio social y productivo; de tal manera que sociabiliza la investigación y producción de conocimiento con perfil tecnológico e innovación social, supeditada a problemáticas más concretas. Esto se da en línea con uno de los objetivos del Ministerio de Ciencia, Tecnología e Innovación (MINCyT), como es el de poner a la investigación al servicio de las necesidades más urgentes de nuestra sociedad. Estos objetivos vinculan lo que es la función de I+D (investigación más docencia), por parte de las universidades con los proyectos del Banco Nacional, en cuanto a su aporte a "la resolución de problemas, necesidades o demandas identificables en la sociedad y/o expresadas por los agentes sociales en la esfera de la política, el mercado, el territorio, la cultura o la estructura social" (Martínez Porta, 2020; Ministerio de Ciencia y Tecnología, 2020).

El vínculo de la innovación universitaria al desarrollo científico-tecnológico y el socio-productivo de un país, se encuentra arraigado a múltiples factores facilitadores u obstaculizadores de dichos desarrollos. Por tanto, resulta menester tener una mirada muldisciplinaria, multifacética, multireferencial de especialistas y desde un abordaje de mayor profundidad. No solo para que quede en un análisis aislado desde una entidad o articulación entre dos actores, como pueden ser empresa-universidad, Estado-empresa, universidad-Estado, entre otros. Para esto, se presenta la inexorable necesidad de atender a un pensamiento articulado desde la universidad, el Estado, las empresas y el resto de las instituciones educativas (tanto del sistema formal como el no formal) o asociaciones no gubernamentales en general (clubes, institutos, colegiaturas, asociaciones, congregaciones, entre otros).

Dentro de las limitantes institucionales para esta vinculación con lo regional, Schwartzman (2006) expone junto con la falta de políticas y normativas adecuadas, la inexistencia de una infraestructura apropiada para el desarrollo de la investigación científica y tecnológica, la escasa cultura y disposición de los académicos universitarios para relacionarse con el sector productivo. En este sentido, la condición en Argentina no es diferente a la descripta, aunque existen escasos estudios dedicados a medir las actividades de vinculación con las universidades argentinas.

\section{Reflexiones comparativas}

Para retomar el objeto de estudio, se puede aseverar que la universidad ha cambiado sustancialmente a lo largo de la historia, desde los rasgos estructurales que la definen como institución como así también en cuanto a su finalidad. El Modo 2 de hacer ciencia (Gibbons et al., 1997) de producción de conocimiento, presenta como rasgos característicos a un conocimiento producido en el contexto de aplicación, la transdiciplinariedad, la heterogeneidad y la diversidad organizativa, la responsabilidad, la reflexividad social y control de calidad. Estos principios enmarcan el camino para una transformación necesaria de los vínculos universitarios en cuanto a la génesis de la ciencia y el medio social.

Estos caracteres traen aparejado una demanda social sobre una nueva función por parte de la universidad, como es la estrecha relación con el sistema productivo específicamente. Bajo esta nueva necesidad, tanto los gobiernos como las personas empresarias y las propias universidades, comenzaron a demandar que estas contribuyeran en forma más activa al desarrollo tecnológico y al crecimiento económico.

Uno de los instrumentos más exitosos para estimular la participación de las universidades hasta ahora, ha sido la creación de las oficinas de transferencia de los resultados de la investigación conocidas como "unidades de vinculación tecnológica" (UVT). Como expone Yamila Kababe (2010, p. 46) las UVT tienen 
como antecedente normativo a la Ley 23.877 en 1990, en la cual se comenta sobre esta estructura como un instrumento formativo para la promoción de la innovación. Esta ley define a las UVT como un "ente no estatal constituido para la identificación, selección y formulación de proyectos de investigación y desarrollo, transmisión de tecnología y asistencia técnica" (art.3 inc. d). Esto se conforma como iniciativa para relacionar la ciencia y la tecnología con la producción, donde las "instituciones oficiales de investigación y desarrollo quedan facultadas para desarrollar unidades de vinculación, con la finalidad de que dispongan de una estructura jurídica que les permita una relación más ágil y contractual con el SP de bienes y servicios" (art. 5). Esta autora ofrece referencia de que los diagnósticos evidencian que la actividad de transferencia tecnológica encomendada a las UVTs sigue siendo una asignatura pendiente.

Las UVTs también se constituyen como difusoras y facilitadoras del trabajo de las empresas, ya que la forma de vinculación de la universidad con la sociedad se da a través del entorno socioeconómico, esto genera un proceso dialógico. Esta relación, como expone Alicia Hernández (2016, p. 33), "implica una relación de reciprocidad, construyendo un concepto más amplio que el de transferencia tecnológica, que es unidireccional entre el que transfiere y el que recibe". Y bajo este concepto, por un lado, las empresas se favorecen con una mayor competitividad y por el otro, las universidades logran integrarse en la sociedad mediante un nuevo contrato social.

Este vínculo educación-empresa debe constituirse como pilar estructural del Estado para la planificación y el desarrollo de la sociedad que se busca alcanzar. Este cimiento contribuye a aprovechar el potencial de creación de empleo, al ofrecer mejores condiciones para un nexo adecuado entre el mundo académico y el laboral, el acceso a empleos de calidad que impulsen el desarrollo de sus habilidades y la generación de nuevo conocimiento (Bonadeo, 2017; Borrego, 2009. La universidad en este lugar, queda con un rol protagónico frente al fortalecimiento del desarrollo social y económico. Por ello, se espera que este lazo se enmarque en políticas y programas de desarrollo social y económico para su concreción práctica y real.

Por otro lado, se considera también relevante pensar en diversas estrategias para el fortalecimiento de estos vínculos, los cuales resulten benéficos a todas las entidades con las que se relaciona la universidad. Como, por ejemplo, poner a funcionar un Consejo Consultivo de Vinculación Social (Cuadriello Olivos, 2008; Garau, 2016; Montoya et al., 2012; Sanz, 2010;) como un ente que aporte asesoramiento para la planificación y la proyección propositiva, desde la transversalidad de políticas y estrategias relacionadas a la solución de problemáticas o satisfacer necesidades emergentes. De esta forma, se fortalece la reciprocidad entre universidad-Estado-sector socio-productivo de una región y, al mismo tiempo, se conforma un proceso de dialogicidad con impacto directo sobre el servicio y el ofrecimiento educativo y profesional en sintonía con las necesidades y las demandas de profesionales, enmarcados en carreras que respondan al desarrollo sustentable de un país.

Además de contemplar un Centro de Estudios Estratégicos (Almuiñas Rivero y Galarza López, 2016; Borrego, 2009) por parte de la universidad, el cual piense en la dirección estratégica integrada por tres componentes de base: el diseño, la implementación y el control de la estrategia. De tal forma que se logre elaborar un diagnóstico desde la complejidad sobre las necesidades y las problemáticas en las diversas áreas del conocimiento.

Como aseveran Almuiñas Rivero y Galarza López (2016) ante un entorno dinámico e incierto como es el actual, la universidad debe desarrollar un papel más activo y transformador para contribuir a superar un conjunto de crisis que impactan negativamente en todos los sectores económicos y sociales, así como en el nivel de vida de la población.

Estas estrategias son acciones poco novedosas en la actualidad, pero se considera menester el reforzamiento como base para una mayor vinculación con el medio social regional y nacional. Como afirma Estébanez (2016) la utilidad social de cualquier conocimiento implica una construcción de poder. Por tanto, el vínculo de la ciencia, la tecnología y la innovación con la atención de los principales problemas de la sociedad no es un logro espontáneo y, para alcanzar este objetivo, debe construirse un espacio 
apropiado en el campo del poder, desde una concepción democrática. Esto implica un proceso participativo de actores del Estado, el sistema ciencia y técnica y la sociedad civil.

Esta concepción democrática se ve reflejada en los discursos actuales frente al debate de los cambios que deben enfrentarse por parte del Estado, la economía y las universidades. Todo lo anterior, se aborda desde la necesidad de redefinir la epistemología institucional de las universidades de una manera más radical y en línea con las modificaciones provenientes de la política pública en su relocalización de recursos orientados a la universidad. Todo esto para incidir directamente sobre la investigación, la innovación y con una vinculación más sólida hacia el mercado (Didriksson Takayanagui, 2020).

A su vez, esto ha replanteado la autonomía de las universidades y su carácter público, en un proceso de altísimo nivel de mercantilización, suscitado en la década del 90. Como expone Axel Didriksson Takayanagui (2016) en sus estudios, "esta tendencia a la mercantilización está impactando de forma desfavorable al entorno de desarrollo de la Educación Superior" (p. 78), lo cual lleva de la autonomía universitaria a su mercantilización como dominio imaginario de la sociedad; de lo educativo como bien público al sentido de bien comercial y de mercancía; de la gestión comunitaria a la gestión global o trasnacional; de la centralidad nacional a la descentralización global y, por último; de la cultura simbólica del espacio al vacío individualista de la competitividad.

Este autor asegura que tal fenómeno se evidencia en países como Japón, Tailandia, Singapur y Malasia (2020), en los cuales se han presentado modificaciones legislativas y normativas para atender el fenómeno descrito, esto ha llevado a que la universidad pública, como se la conoce, haya desaparecido al encontrarse en una relación directamente ligada con sus funciones volcadas al mercado.

Esta lógica de relación "Estado, mercado y universidad", ha reorientado los recursos del Estado hacia las localidades, la innovación y la investigación científica-tecnológica de las principales universidades. Esto generó un fortalecimiento del mercado local, regional y la perspectiva que han llevado a copar los mercados internacionales sobre todo de innovación tecnológica.

Este fenómeno lleva a replantear la definición epistemológica de la universidad, con el fin de incorporar lo que puede denominarse como calidad social de la Educación Superior. Esto en respuesta natural al desarrollo de la universidad, ante la incorporación del concepto de responsabilidad social en sus funciones. Lo anterior, ha llevado a las instituciones a obtener sus productos y resultados con un fuerte componente de consciencia y acción social. En concordancia con ello, las investigaciones han comenzado a valorarse en virtud de la vinculación que tienen con el medio y ya no solo en la cantidad y calidad de producción. Un ejemplo de esto, es el Consorcio de países escandinavos (Dinamarca, Noruega y Suecia) (Pedersen, 1979) y la Liga europea de universidades de investigación, las cuales poseen una redefinición de sus criterios de investigación y la epistemología institucional. La idea es que sus productos, resultados y mecanismos de egreso tengan una directa vinculación con la sociedad bajo el concepto de calidad social (Pérez, 2002; Somarriba Arechavala, 2010).

Lo que se busca es que el Estado se vuelque al trabajo, en conjunto con las universidades ante los cambios de modelos que enfrentan estas entidades. Como es en la interculturalidad, la innovación con carácter social, la incidencia de la investigación en el mejoramiento de las poblaciones, la reestructuración y la reorganización radical de los términos en los cuales las universidades deben asumir su autonomía, en la justa medida en que estas protagonicen su responsabilidad social (Didriksson Takayanagui, 2020).

En cuanto a Latinoamérica, estos cambios radicales también se evidencian al redefinirse las normativas. Se evidencia la distinción del concepto de universidad entre los países de corte neoliberal vs. gobiernos que poseen mayores estructuras con vinculación a lo social. Donde los últimos, establecen vínculos que privilegian la inclusión social en beneficio de las comunidades, junto con iniciativas que mantienen la gratuidad como es en las universidades argentinas. 
Ante estos cambios tan vertiginosos sobre la definición y, por lo tanto, de las funciones inherentes a las universidades, cabe preguntarse si existe la universidad tal como se la conocía antiguamente. Mucho se ha argumentado sobre que la universidad sigue manteniendo principios tradicionales y medievales como referentes de la organización de la vida académica; entre los cuales encontramos la autonomía universitaria, la consciencia crítica, el estudio desde la perspectiva de disciplinas y la investigación lineal. Pero también se ha aseverado que estos principios en la realidad de las estructuras modernas de la universidad, en la gestión del conocimiento y sus estructuras curriculares, ya no son las mismas e incluso esa universidad sigue manteniendo principios antiguos, viejos, ancestrales, pero indefectiblemente ya es otra universidad.

Se devela un modelo distinto de universidad, en el cual predomina la función de investigación e innovación, la interdisciplinariedad, la transdisciplinariedad, la complejidad, la articulación de saberes, en donde prima el concepto de calidad social o el de incidencia de la vinculación con la sociedad, en muchos casos de manera dual. Tanto como de beneficio privado y como de bien público, en donde la administración y la burocracia ha tenido un extraordinario proceso de expansión, que ha significado un fortalecimiento de estructuras burocráticas y administrativas por encima de la vida académica.

De igual manera, el tema de la investigación no ha repercutido sustancialmente como se esperaba en las universidades. Por lo tanto, en la universidad se sigue teniendo una gran carga sobre la docencia y, por lo tanto, todo lo que es el conocimiento moderno, la vinculación y la incidencia de estos saberes hacia las comunidades sigue siendo insuficiente, al seguir circunscripto a la formación del profesional que egresa. En consecuencia, la inversión en ciencia y tecnología se perpetúa en la deficiencia. Y en este sentido, América Latina es la región que menos ha contribuido en generar conocimiento, su incidencia en la sociedad y el desarrollo.

\section{SÍNTESIS Y REFLEXIONES FINALES}

Frente a una transformación continua y dinámica de las estructuras configurantes de la universidad como se la conoce, da lugar a un proceso emergente frente a los cambios sociales y culturales que demandan otras funciones y acciones de las instituciones educativas, las cuales se hallan enmarcadas en un contexto político, social y económico; donde indefectiblemente la universidad-además de buscar su propio financiamiento-, debe vincularse por medio de la empatía y la responsabilidad social. Ya no puede quedarse en un lugar de elitismo frente al clasismo social que representa la academia.

La sociedad demanda una universidad mucho más arraigada a las necesidades. Ya no solo desde un sector poblacional, sino desde la totalidad de las clases, debe configurar un sistema que realmente contemple al "otro" como un par en la igualdad de condiciones y posibilidades. Pero desde un posicionamiento distinto al concepto de inclusión o integración social, como se recurre habitualmente en la retórica discursiva.

Esto también llevará a cambiar el concepto de "gratuidad" frente al acceso y permanencia de las trayectorias, como expone Fernández Lamarra (2020), cuando se piensa en un sistema de mayor equidad frente a las posibilidades individuales y distintivas de clases. La heterogeneidad se expresa en los altos grados de inclusión educativa, la alta tasa de desgranamiento de la matrícula y los altos grados de segmentación de la oferta.

Son diversos los cambios y desafíos que enfrentan y enfrentarán las universidades, pero se tiene la certeza de que la definición, la estructura y las funciones institucionales mutarán en una ajustada vinculación con el medio en que se encuentre. El concepto de autonomía también se verá modificado, transformándose sin desaparecer, en un concepto con un profundo sentir sobre la responsabilidad social por parte de la universidad. Se toma la palabra de Norberto Fernández Lamarra (2020), al determinar que las 
políticas necesariamente deben estar pensadas desde la planificación de programas y proyectos; pues es la manera para que bajen a la realidad concreta en la satisfacción de necesidades. Para esto, la planificación estratégica es clave, para establecer las pautas de innovación junto con un seguimiento de esos proyectos por parte de la universidad.

Este autor, Fernández Lamarra, realiza una crítica a la universidad de América Latina que sigue siendo hasta la actualidad, sobre lo poco innovadora tanto en lo institucional como en lo organizativo. La poca innovación existente en general, además que no se registra sistemáticamente, no se evalúa y tampoco difunde. Pareciera que esta problemática no se presenta como un tema de investigación por parte de las universidades, por lo tanto, se sabe poco al respecto.

Por otro lado, la dura paradoja que deviene de los grandes avances en ciencia y tecnología, es que la pobreza y la exclusión siguen avanzando, se profundizan las desigualdades sociales y territoriales. Esta problemática se evidencia y fortalece a la par con el crecimiento y el desarrollo de la educación en todos sus niveles.

Así como la situación de vida actual frente a una pandemia provocada por el virus responsable de la COVID-19, presenta consecuencias adversas como el cierre de escuelas, altos costos sociales y económicos para todas las comunidades. Su impacto particularmente severo para la infancia más vulnerable y marginada. Estas interrupciones resultantes exacerban las disparidades ya existentes dentro del sistema educativo, pero también en otros aspectos de la vida como: el aprendizaje interrumpido; la nutrición deficiente; confusión y estrés para las personas docentes; progenitores sin preparación para la educación a distancia y en el hogar; desafíos para crear; mantener y mejorar la educación a distancia; brechas en el cuidado de la niñez; costos económicos elevados; tensión involuntaria en los sistemas de salud; mayor presión sobre las escuelas y los sistemas escolares; aumento de la tasa de deserción escolar; mayor exposición a la violencia y la explotación y el aislamiento social (UNESCO, 2020).

Todo lo anterior conlleva a la necesidad de una nueva articulación de vida que "presupone un giro epistemológico, cultural e ideológico que respalde las soluciones políticas, económicas y sociales que garanticen la continuidad de una vida humana digna en el planeta" (De Sousa Santos, 2020, p. 84). Por lo tanto, todo cambio, debe emerger desde las instituciones educativas como aporte a la construcción o reconstrucción de la sociedad anhelada.

La evidencia está al alcance de toda la sociedad. Los desafíos que emergen ante la necesidad de respuestas y soluciones, deben surgir desde los sectores de poder y decisión como son el Estado y la universidad. Ya no se puede mirar hacia otro lado, ante un Estado ausente o poco efectivo, la universidad debe entrar en acción frente a las demandas sociales, desde su lugar de poseedora del conocimiento científico-especializado y su función primaria de servicio al medio social.

La situación apremiante actual de una profunda crisis social, económica y sanitaria, lleva ante la inaplazable necesidad de pensar y repensar en la generación de políticas desde la gestión universitaria, las cuales le ofrezcan centralidad y sustento al conocimiento para el desarrollo y el acompañamiento actual y futuro de la sociedad. Todo esto, por medio de la innovación, la tecnología y la transferencia de la ciencia con el medio socio productivo desde una profunda convicción de responsabilidad y calidad social universitaria.

La mira debe posicionarse en el combatir a la desigualdad, la inequidad y la lucha por la justicia social, acompañando al Estado y al medio socio productivo, desde el aporte de soluciones con una mirada responsable socialmente. Es perentorio que ofrezca una educación real de calidad que articule a todos los niveles educativos, dé respuestas al contexto productivo por medio de un fuerte entretejido de sus trazos vinculares hacia la mayor cantidad de dimensiones en las que se pueda acceder desde la investigación. Con un constante sentir comprometido sobre la proyección de un futuro con mayor paridad, justicia y búsqueda de la felicidad como objetivos de desarrollo sustentable de una nación. 
Abásolo, M. A. L. (2008). La gestión del conocimiento y la universidad del futuro. Revista Faces, 17(1):21-34.

Academia Nacional de las Ciencias. (2020, setiembre). Breve reseña de la creación de la Academia. Recuperado de Academia Nacional de las Ciencias: https://www.anc-argentina.org.ar/es/ institucional/breve-resena-de-la-creacion-de-la-academia/

Acosta, C. A. P. (2011). La gestión del conocimiento en las universidades como baluarte organizacional. InterSedes, 12(23).

Almuiñas Rivero, J. L., y Galarza López, J. (2016). Dirección estratégica y gestión de riesgos en las universidades. Revista Cubana de Educación Superior, 35(2):83-92.

Alonso-Arévalo, J. (2007). Gestión de la Información, gestión de contenidos y conocimiento.

Armengol, M. C. (2005). Nueva universidad ante la sociedad del conocimiento. RUSC. Universities and Knowledge Society Journal, 2(2):1.

Balderas, R. (2009). ¿Sociedad de la información o sociedad del conocimiento?. El cotidiano, (158):75-80.

Böhme, E. (recuperado 2021, 4 de marzo). Historia de la Universidad - una descripción general. GeorgAugust-Universität-Göttingan. Recuperado de: https://www.uni-goettingen.de/en/history+of+t he+university+\%e2\%80\%93+an+overview/90607.htm

Bonadeo, D. J. (2017). Análisis de la implementación del Programa de fortalecimiento de las oficinas de Vinculación y Transferencia Tecnológicas (OVTT) del Ministerio de Ciencia, Tecnología e Innovación productiva de Argentina, en el marco de las políticas de promoción de la innovación: el caso de la OVTT mendocina Argentec SRL.

Borrego, A. A. (2009). Vinculación universidad-empresa y su contribución al desarrollo regional. Ra Ximhai: Revista científica de sociedad, cultura y desarrollo sostenible, 5(3):407-414.

Bory, E. P., y Ramírez, B. I. (2002). Sociedad del Conocimiento.

Burch, S. (2005). Sociedad de la información/Sociedad del conocimiento. Palabras en juego, 54-78.

Cabero Almenara, J. (2008). La formación en la sociedad del conocimiento. Indivisa: Boletin de Estudios e lnvestigación, 10:13-47.

Canturelli, A. (1964). La Universidad. Su esencia, su vida, su ambiente. Córdoba: Dirección General de Publicaciones de la Universidad Nacional de Córdoba.

Carranza, C. C. (2007). Educacion para una sociedad del conocimiento.

Chudnovsky, D., y López, A. (1995). Política tecnológica en la Argentina: hay algo más que laissez faire?. CENIT (Centro de Investigaciones para la Transformación).

Clark, B. R. (1991). El sistema de educación superior. Una visión comparativa de la organización académica. México DF, México: Nueva Imagen.

Comisión Europea. (2012, 11 de octubre). Reportaje - Alemania, un líder mundial en tecnología, ingeniería e innovación. Recuperado de: https://cordis.europa.eu/article/id/89457feature-stories-germany-a-world-leader-in-technology-engineering-and-innovation/es

Coronado, G. (2005). De Otto Hahn al Proyecto Manhattan: Señalamientos sobre un desarrollo científico-tecnológico. Energía y tecnología nuclear: discusiones éticas, sociales y ambientales, (19).

Cuadriello Olivos, H. (2008). Neoindigenismo y participación ciudadana: el Consejo Consultivo de la CDIy la participación indígena (Master's thesis, México: FLACSO, Sede Académica de México).

Derisi, O. (1980). Naturaleza y vida de la universidad. Buenos Aires: El Derecho-UCA. 
De Sousa Santos, B. (2020). La cruel pedagogía del virus. CLACSO. Buenos Aires. Recuperado de: http:// biblioteca.clacso.edu.ar/clacso/se/20200430083046/La-cruel-pedagogia-del-virus.pdf

Didriksson Takayanagui, A., Herrera Márquez, A., Villafán Aguilar, L., Huerta Martínez, B. y Torres Ríos, D. (2016). De la privatización a la mercantilización de la educación superior. Universidad Nacional Autónoma de México. Instituto de Investigaciones sobre la universidad y la Educación. México. Recuperado de: http://132.248.192.241/ editorial/wp-content/uploads/2016/06/de-la-privatizacion-a-la-mercantilizacion-de-la-educacion-superior.pdf

Didriksson Takayanagui, A. (2020, 6 de febrero). Tendencias de cambio en la Universidad actual: un panorama comparado y regional. Webinar UNESCO IESALC. Instituto de Investigaciones sobre la Universidad y la Educación (IISUE). UNAM. México. Recuperado de: https://www.youtube.com/ watch?v=X7UIG7h5QVc

Dini, M., Stumpo, G. e Italiana, I. C. (2011). Políticas para la innovación en las pequeñas y medianas empresas en América Latina.

Estébanez, M. E. (2016). Ciencia, Universidad y entornos. Conceptos y reflexiones. Centro Redes / CONICET y Universidad de Buenos Aires, Facultad de Ciencias Sociales. Publicado en la Revista: Política Universitaria, Nro. 3 sept 2016, Bs As. EditoriallEC/Conda.

Fainholc, B. (2006). Rasgos de las universidades y de las organizaciones de educación superior para una sociedad del conocimiento, según la gestión del conocimiento. RUSC. Universities and Knowledge Society Journal, 3(1).

Farfán Buitrago, D. Y., y Garzón Castrillón, M. A. (2006). La gestión del conocimiento. Argentina: Editorial Universidad del Rosario.

Fernández Lamarra, N. (2020, 13 de febrero). Universidad, innovación y futuro en América Latina y el Caribe. Webinar UNESCO IESALC. Universidad Tres de Febrero (UNTREF). Buenos Aires. Recuperado de: https://www.youtube.com/watch?v=pEH5JgiS-v0\&t=903s

Ferraro, C. A. y Goldstein, E. (2011). Políticas de acceso al financiamiento para las pequeñas y medianas empresas en América Latina.

Fundación Metropolitana. (2014, agosto). El crecimiento de los parques industriales, motor de la economía para la región metropolitana. Obtenido en Fundación Metropolitana. Planificación y participación para la gran Buenos Aires: http://metropolitana.org.ar/idm/el-crecimiento-de-losparques-industriales-motor-de-la-economia-para-la-region-metropolitana/

Garau, A. (2016). Consejo Consultivo de la Escuela Argentina de Negocios: una experiencia de aprendizaje colaborativo entre la Universidad y el sector productivo. Revista Argentina de Investigación en Negocios, 2(1-2):71-78.

García Aretio, L. (2012). Sociedad del conocimiento y educación.

García-Peñalvo, F. J. (2014). Formación en la sociedad del conocimiento, un programa de doctorado con una perspectiva interdisciplinar. Teoría de la Educación. Educación y Cultura en la Sociedad de la Información, 15(1):4-9.

Gibbons, M., Limoges, C., Nowotny, H., Schwartzman, S., Scott, P. y Trow, M. (1997). La nueva producción del conocimiento. La dinámica de la ciencia y la investigación en las sociedades contemporáneas, 121.

Hernández, A. (2016). Las Unidades de Vinculación Tecnológica (UVT) en las universidades públicas argentinas: El caso de la Universidad Nacional de Mar del Plata (Doctoral dissertation, Universidad Nacional de Mar del Plata. Facultad de Ingeniería. Argentina).

Inteligencia Jurídica. (2020, 3 de setiembre). Legislación. Se crea el Programa Nacional para el Desarrollo de Parques Industriales. Obtenido de Microjuris.com - AL Día ARGENTINA: https://aldiaargentina. microjuris.com/2020/09/03/legislacion-se-crea-el-programa-nacional-para-el-desarrollo-de-parques-industriales/ 
Jacovkis, M. (2019). Seminario de Política y Gestión de Ciencia y Tecnología en las universidades. Doctorado en Política y Gestión de la Educación Superior. UNTREF.

Jover, J. N., Montalvo, L. F., y Ones, M. S. I. P. (2006). La gestión del conocimiento, la ciencia, la tecnología y la innovación en la nueva universidad: una aproximación conceptual. La Nueva Universidad Cubana y su contribución a la universalización del conocimiento, 5.

Kababe, Y. (2010). Las Unidades de Vinculación Tecnológica y la articulación entre el sector científico tecnológico y el sector empresario. SaberEs. (2):41-58.

Krüger, K. (2006). El concepto de sociedad del conocimiento. Revista bibliográfica de geografía y ciencias sociales, 11(683):1-14.

Lanzafame, S. (2019, 5 de mayo). ¿Cómo se iniciaron los parques industriales?. Obtenido de Conexión de Parques. Lo que pasa en la industria: https://conexionparques.com.ar/ como-se-iniciaron-los-parques-industrial

López-Muñoz, F. (2020, 3 de mayo). Segunda guerra mundial: cómo los atroces experimentos de los nazis dieron lugar al nacimiento de la bioética. BBC News Mundo. Obtenido de: https://www.bbc.com/ mundo/noticias-52501865

Luque Cózar, R. y Domínguez López, J. J. (2009). El proyecto Manhattan: un ejemplo de producción desde la universidad.

Mateo, J. L. (2006). Sociedad del conocimiento. Arbor, 182(718):145-151.

Maroto Barquero, C. (1989). La influencia alemana en el desarrollo de la geopolítica a mediados del siglo XX. Revista Estudios. ISSN-e 1659-3316, ISSN 1659-1925. Recuperado de: https://dialnet.unirioja.es/servlet/tesis?codigo $=91958$

Martínez Porta, L. (2020). Sobre la visibilización de la investigación con impacto tecnológico y social. Carlos Marquis (ed.). La agenda universitaria V. Criterios y propuestas de gestión y políticas universitarias. Buenos Aires: Universidad de Palermo, colección de Educación Superior.

Millán, J. A. (2001). La lectura y la sociedad del conocimiento. Madrid: Federación de Gremios de Editores de España.

Ministerio de Educación, Ciencia y Tecnología. (2006). Programa de Modernización tecnológica III. Reglamento Operativo. Secretaría de ciencia, tecnología e innovación productiva. Buenos Aires. Recuperado de: https://www.argentina.gob.ar/sites/default/files/foncyt-reglamento_operativo.pdf

Ministerio de Ciencia y Tecnología. (2020). Banco Nacional de Proyectos de Desarrollo Tecnológico y Social. Secretaría de Articulación Científico Tecnológica. Buenos Aires. http:/www. Argentina.gob.ar.

Ministerio de Justicia y Derechos Humanos. (1990). Ley № 23877. Promoción y fomento de la innovación tecnológica. Presidencia de la Nación. InfoLeg Información Legislativa. Argentina. Recuperado de: http://servicios.infoleg.gob.ar/infolegInternet/anexos/0-4999/277/norma.htm

Montoya, M. M. A. C. S., Montoya, M. J. P. S. y Montoya, M. A. M. S. (2012). La participación social mediante un Consejo Consultivo, elemento necesario para elevar la Calidad Integral de la Educación Superior en el Estado de Nayarit (Caso: Universidad Autónoma de Nayarit). México: XV Congreso Internacional sobre Innovaciones en Docencia e Investigación en Ciencias Económico Administrativas.

Moreno, I. F. (2009). La sociedad del conocimiento. Revista Científica General José María Córdova, 5(7):40-44.

Morin, E. y Ruíz, J. L. S. (2005). Con Edgar Morin, por un pensamiento complejo (Vol. 22). Ediciones AKAL.

Muscará, F. (2014). Pasado, presente y futuro de la Educación Superior. Argentina: Editorial de la Universidad Aconcagua.

Pablos Pons, J. (2010). Universidad y sociedad del conocimiento. Las competencias informacionales y digitales. RUSC. Universities and Knowledge Society Journal, 7(2). 
Pascolini, A. (2012). Historia Nuclear. NUPEX (Nuclear Physics Experience). Obtenido de: http://nupex. eu/index.php?lang=es\&g=textcontent/nuclearhistory/nuclearhistory

Pastor, J. (2018). Los grandes avances tecnológicos que nos dejó la Primera Guerra Mundial. XATAKA. Obtenido de: https://www.xataka.com/historia-tecnologica/ los-grandes-avances-tecnologicos-que-nos-dejo-la-primera-guerra-mundial

Pedersen, M. (1979). La transformación de las Universidades Escandinavas: Experimentos de participación, politización y otras discontinuidades. Dialnet. Recuperado de: https://dialnet.unirioja.es

Pérez, C. (2002). La calidad social. Entre la complejidad y la viabilidad de su análisis. Observatorio Medioambiental, 5:217-228. Recuperado a partir de https://revistas.ucm.es/index.php/OBMD/ article/view/OBMD0202110217A

Pérez Lindo, A. (1985). Universidad, política y sociedad. Buenos Aires: EUDEBA S.E.M.

Pérez Lindo, A. (2014). Gestión del Conocimiento en La Universidad. Eje Temático: Gobierno y Poder en la Universidad. IV Encuentro Nacional y Latinoamericano de Educación.

Rodríguez Castellanos, A., Araujo de la Mata, A. y Urrutia Gutiérrez, J. (2001). La gestión del conocimiento científico-técnico en la universidad: un caso y un proyecto.

Somarriba Arechavala, M.N. (2010) "Aproximación a la medición de la Calidad de Vida Social e Individual en la Europa Comunitaria", Edición electrónica gratuita. Texto completo en www.eumed.net/ tesis/2010/mnsa/

Sábato, J. (2011). El Pensamiento Latinoamericano en la Problemática Ciencia-tecnología-desarrollodependencia. Argentina: Colección Placted.

Sábato, J. A. y Botana, N. R. (1970). La ciencia y la tecnología en el desarrollo futuro de América Latina.

Salazar-Gómez, E. y Tobon, S. (2018). Análisis documental del proceso de formación docente acorde con la sociedad del conocimiento. Espacios, 39(45):17.

Santos, X. G. (2018). La historia (más conflictiva) del PC. Obtenido de MeriStation: https://as.com/meristation/2018/11/10/reportajes/1541863702_091383.html

Sanz, M. J. G. (2010). Actividad del Consejo Consultivo de Castilla-La Mancha en materia constitucional y estatutaria. Parlamento y Constitución. Anuario, (13):425-442.

Schwartzman, S. (2006). América Latina Universidades en transición. Organización de los Estados Americanos, Washington: Colección Interamer, nº 6.

Tobón, S., Guzmán, C. E., Silvano Hernández, J. y Cardona, S. (2015). Sociedad del conocimiento: estudio documental desde una perspectiva humanista y compleja. Paradigma, 36(2):7-36.

Tognetti, L. y Page, C. A. (2000). La Academia Nacional de Ciencias: etapa fundacional, siglo XIX. Academia Nacional de Ciencias.

Tubella, I. y Requena, J. V. (2005). Sociedad del conocimiento. Editorial UOC.

UNESCO. (2010). Declaración Universal de la UNESCO sobre la diversidad cultural. PRAXIS, 64:65.

UNESCO.(2020).COVID-19.Obtenido de LaUNESCO:https://en.unesco.org/covid19/educationresponse/ consequences

Venturuzzi, G., Longo, E., Martín, M. y Velazco, E. (2007). Incubadoras y Parques Tecnológico. Observatorio de Políticas Públicas cat. Opp/cag/2007-08. Coordinación General del Cuerpo de Administradores Gubernamentales Jefatura de Gabinete de Ministros.

Vergara Aimone, J. A. (1997). El Proyecto Manhattan II PARTE. Chile: Revista Marina. 